\title{
High plasma concentration of beta-D-glucan after administration of sizofiran for cervical cancer
}

This article was published in the following Dove Press journal:

International Journal of General Medicine

4 September 2010

Number of times this article has been viewed

\author{
Hirokazu Tokuyasu' \\ Kenichi Takeda' \\ Yuji Kawasaki' \\ Yasuto Sakaguchi ${ }^{2}$ \\ Noritaka Isowa ${ }^{2}$ \\ Eiji Shimizu ${ }^{3}$ \\ Yasuto Ueda ${ }^{3}$ \\ 'Divisions of Respiratory Medicine \\ ${ }^{2}$ Thoracic Surgery, Matsue Red \\ Cross Hospital, 200 Horomachi, \\ Matsue, Shimane; ${ }^{3}$ Division of Medical \\ Oncology and Molecular Respirology, \\ Department of Multidisciplinary \\ Internal Medicine, Faculty of Medicine, \\ Tottori University, Yonago, Japan
}

Correspondence: Hirokazu Tokuyasu

Division of Respiratory Medicine,

Matsue Red Cross Hospital,

200 Horomachi, Matsue,

Shimane 690-8506, Japan

Email tokuun3200@yahoo.co.jp
Abstract: A 69-year-old woman with a history of cervical cancer was admitted to our hospital for further investigation of abnormal shadows on her chest roentgenogram. Histologic examination of transbronchial lung biopsy specimens revealed epithelioid cell granuloma, and Mycobacterium intracellulare was detected in the bronchial lavage fluid. The plasma level of $(1 \rightarrow 3)$-beta-D-glucan was very high, and this elevated level was attributed to administration of sizofiran for treatment of cervical cancer 18 years previously. Therefore, in patients with cervical cancer, it is important to confirm whether or not sizofiran has been administered before measuring $(1 \rightarrow 3)$-beta-D-glucan levels.

Keywords: $(1 \rightarrow 3)$-beta-D-glucan, cervical cancer, Mycobacterium intracellulare, sizofiran

\section{Introduction}

$(1 \rightarrow 3)$-beta-D-glucan is a characteristic cell wall component of almost all fungi. The measurement of the plasma concentration of this glucan is clinically useful when screening for invasive fungal infections or fungal febrile episodes. ${ }^{1}$ In addition, this glucan is known to stimulate humoral and cell mediated immunity in humans, and has antitumor effects. ${ }^{2}$ High plasma concentrations of $(1 \rightarrow 3)$-beta-D-glucan may occur even in the absence of a fungal infection because of the administration of sizofiran, an antitumor $(1 \rightarrow 3)$-beta-D-glucan preparation (SPG; Kaken Pharmaceuticals, Tokyo, Japan). We report an extremely rare case of a patient with elevated plasma concentrations of $(1 \rightarrow 3)$-beta-D-glucan 18 years after she was treated with sizofiran for cervical cancer.

\section{Case report}

A 69-year-old woman was admitted to our hospital in November 2007 for further examination of abnormal shadows in both lungs. She was a nonsmoker and had no history of occupational exposure. She had undergone hysterectomy in 1990 and was administered SPG for six months thereafter. Physical examination revealed that she had no skin lesions or neurologic abnormalities. There was no peripheral lymphadenopathy in the cervical, axillary, or inguinal region. Fine crackles were audible in the middle lung fields bilaterally.

The white blood cell count was $6.2 \times 10^{9} / \mathrm{L}$, with $56.6 \%$ neutrophils, $34.3 \%$ lymphocytes, $5.2 \%$ monocytes, $3.2 \%$ eosinophils, and $0.7 \%$ basophils. The level of C-reactive protein was normal. Her fasting blood glucose level was $149 \mathrm{mg} / \mathrm{dL}$ and $\mathrm{Hb}_{\mathrm{Alc}}$ was $6.8 \%$. The serum concentration of immunoglobulin $\mathrm{G}$ (IgG) was elevated 
to $2034 \mathrm{mg} / \mathrm{dL}$ (normal, 837-1825 $\mathrm{mg} / \mathrm{dL}$ ), and the serum IgM and IgA levels were within the normal range. The level of $(1 \rightarrow 3)$-beta-D-glucan was elevated to $260 \mathrm{pg} / \mathrm{mL}$ (cutoff value, $11 \mathrm{pg} / \mathrm{mL}$ ) and the plasma endotoxin level was normal. Aspergillus antigens (Platelia ${ }^{\circledR}$ Aspergillus EIA; Bio-Rad, Marnes-la-Coquette, France) and Candida antigens (CandTec; Ramco Laboratories, Inc., Houston, TX.) were absent. There was no liver and kidney dysfunction. A chest roentgenogram showed infiltrative shadows in both lungs (Figure 1). Chest computed tomography revealed centrilobular nodules and patchy infiltrative shadows. Bronchiectasis was observed in the upper lobes of both lungs and in the middle lobe of the right lung (Figure 2). There was no radiographic evidence of infection, purulence, or tumors of the abdominal or pelvic organs. The areas of intense fluorodeoxyglucose activity detected by ${ }^{18}$ FDG-positron emission tomography coincided with the infiltrative shadows detected by computed tomography. Histologic examination of transbronchial lung biopsy specimens obtained from the right upper lobe revealed epithelioid cell granuloma (Figure 3). Mycobacterium intracellulare was detected in the bronchial lavage fluid, but Aspergillus spp, Candida spp, and Pneumocystis jiroveci were not detected. There was no evidence of systemic fungal infection in this patient. Therefore, the patient was diagnosed with pulmonary $M$. intracellulare infection. However, the cause of the high plasma concentration of $(1 \rightarrow 3)$-betaD-glucan remained unknown. Because the possibility of

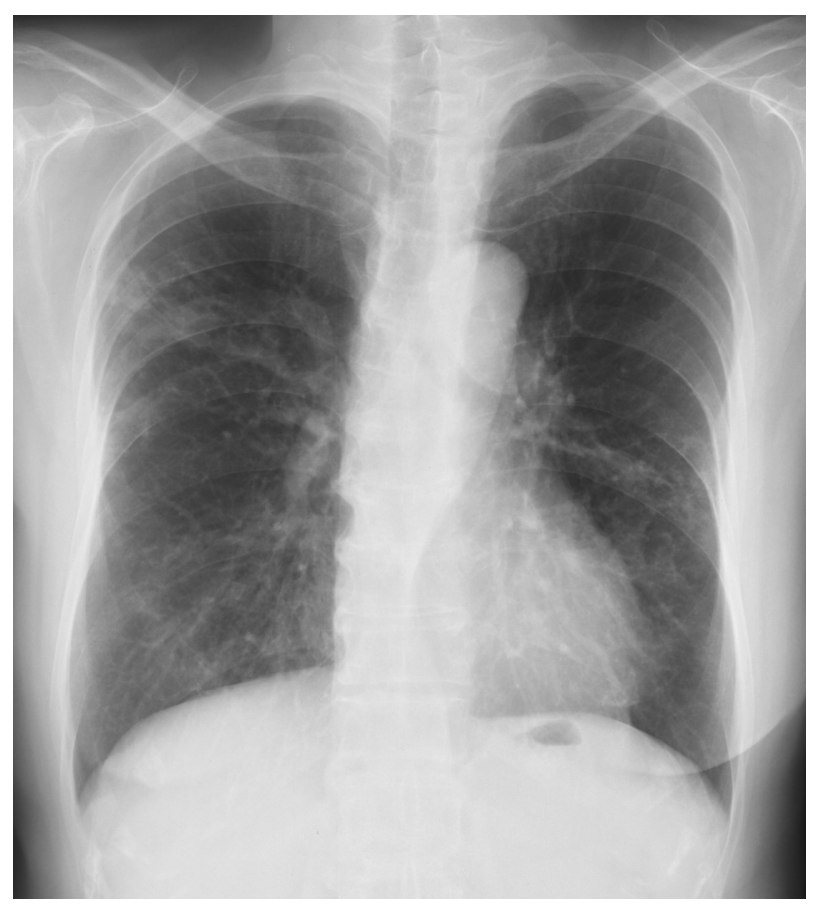

Figure I Chest roentogenogram showing infiltrative shadows in both lungs.
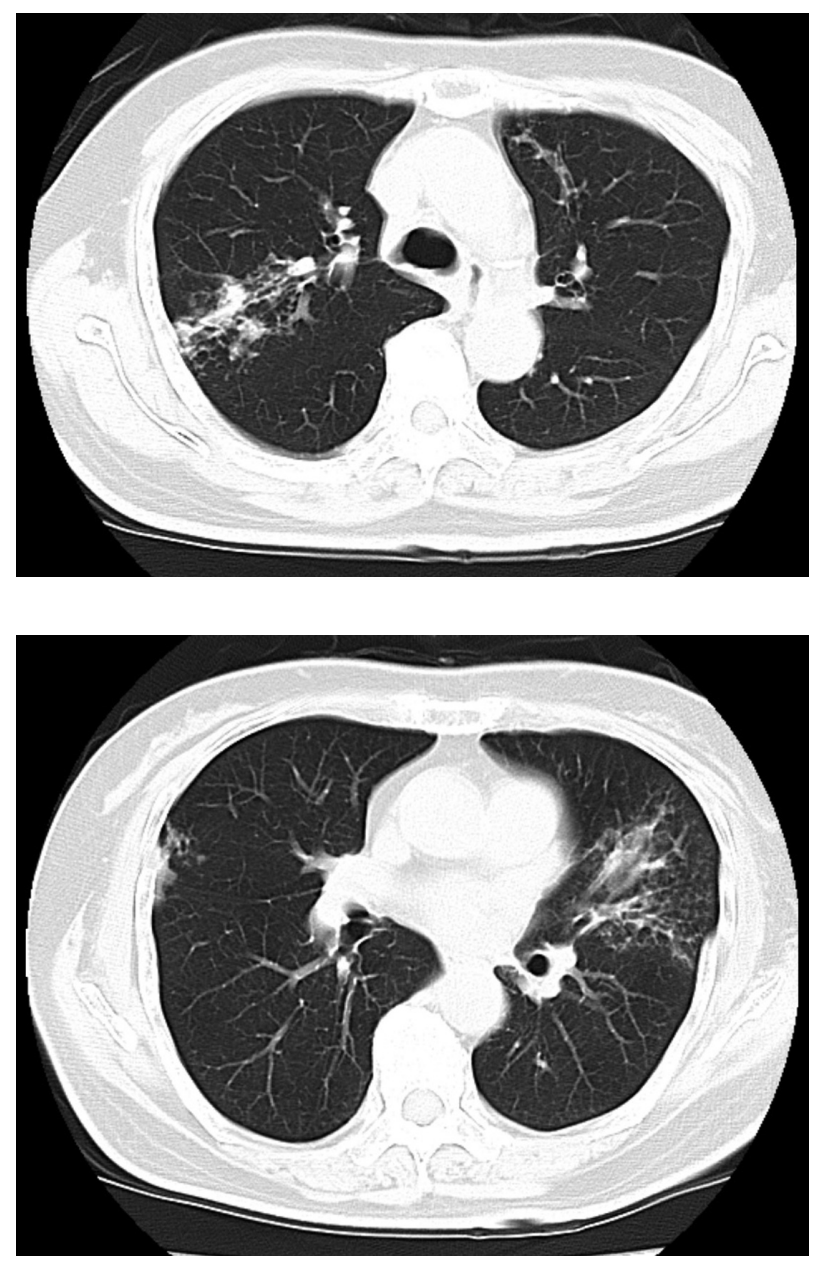

Figure 2 Chest computed tomography showing centrilobular nodules and patchy infiltrative shadows and bronchiectasis in the upper lobes of both lungs and the middle lobe of the right lung.

deep-seated mycosis could not be ruled out, the patient was treated with oral voriconazole for three months. However, the plasma concentration of $(1 \rightarrow 3)$-beta-D-glucan remained high; by February 2009, it had increased from 160 pg/mL to $410 \mathrm{pg} / \mathrm{mL}$ (Figure 4). Because it was unlikely that any other factor was responsible, we concluded that the high $(1 \rightarrow 3)$-beta-D-glucan level was caused by intramuscular injections of $40 \mathrm{mg}$ SPG every week for six months, which had been administered for the treatment of cervical cancer 18 years previously.

\section{Discussion}

The plasma concentration of $(1 \rightarrow 3)$-beta-D-glucan, a characteristic cell wall component of almost all fungi and absent in bacteria, is widely used in Japan as an indicator of fungal infection. In 2004, the United States Food and Drug Administration recognized that measurement of this glucan is useful for the detection of fungal infection. 


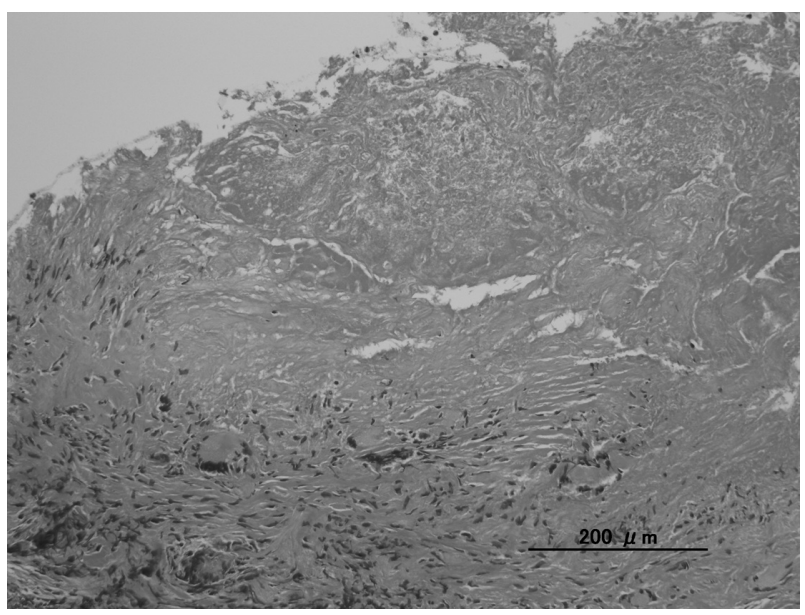

Figure 3 Histologic examination of hematoxylin-eosin stained transbronchial lung biopsy specimens obtained from the right upper lobe revealed caseous necrosis with lymphocytes, epithelioid granuloma, and Langhans' giant cells.

Factor $\mathrm{G}$ in the lysate from the Japanese horseshoe crab (Tachypleus tridentatus) reacts with $(1 \rightarrow 3)$-beta-D-glucan [3]. Obayashi et al reported that the determination of plasma $(1 \rightarrow 3)$-beta-D-glucan concentration for the detection of fungal infection with a cutoff value of $20 \mathrm{pg} / \mathrm{mL}$ has a sensitivity of $90 \%$ and a specificity of $100 \% .^{1}$ In Japan, three test kits are currently available for the measurement of plasma glucan levels, ie, the FUNGITEC G Test MK (FUNGITEC-MK), beta-Glucan Test Wako (Wako), and beta-Glucan Test Maruha (Maruha). The FUNGITEC-MK kit, which involves alkaline pretreatment of the sample before measurement, is widely used in Japan because it is highly sensitive and enables easy handling of many samples. In our hospital, we measure glucan levels using the Wako kit, which shows an overall agreement rate of nearly $90 \%$ with the FUNGITEC-MK kit for fungal infection. ${ }^{4}$ However, in cases where $(1 \rightarrow 3)$-beta-D-glucan preparations, such as SPG and lentinan, have been administered, alkaline pretreatment of the sample (FUNGITEC-MK kit) yields drastically higher beta-D-glucan values than the values obtained by dilution and heat pretreatment (Wako and Maruha kits). ${ }^{5}$ SPG and lentinan have a triple helical conformation that is denatured into a single helical structure under alkaline conditions. ${ }^{6,7}$ Most of the $(1 \rightarrow 3)$-beta-D-glucan in the blood of patients with deep-seated mycosis is in the single helical conformation, which reacts more strongly with factor $\mathrm{G}$ than the triple helical conformation of beta-D-glucan. ${ }^{5}$ In December 2008, the level of $(1 \rightarrow 3)$-beta-D-glucan in our patient was found to be $3240 \mathrm{pg} / \mathrm{mL}$ (cutoff value, $20 \mathrm{pg} / \mathrm{mL}$ ) with the FUNGITEC-MK kit and $124 \mathrm{pg} / \mathrm{mL}$ (cutoff value, $11 \mathrm{pg} / \mathrm{mL}$ ) with the Maruha kit, whereas the glucan level was found to be $268 \mathrm{pg} / \mathrm{mL}$ with the Wako kit.

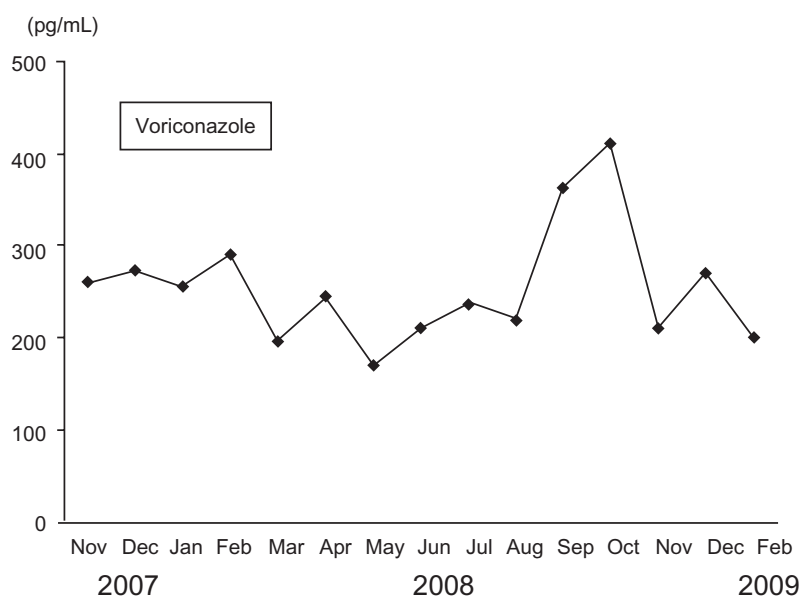

Figure 4 Plasma concentration of beta-D-glucan between November 2007 and February 2009

These results suggested that the administered SPG remained in the triple helical conformation, and reacted strongly with factor $\mathrm{G}$ after alkaline treatment.

Some clinical conditions interfere with the results obtained using the Wako kit. False positive reactions have been reported in patients receiving human immunoglobulin products, which contain substances that react with factor G. ${ }^{8}$ These substances include drugs containing $(1 \rightarrow 3)$-betaD-glucan, for example, lentinan and SPG,, 10 the cellulose membrane used for hemodialysis, ${ }^{11}$ and glucan-containing gauze used in surgery. ${ }^{12}$ However, in the case of our patient, there was no history of the use of glucan-containing gauze, intake of Chinese medicines, ${ }^{13}$ supplements, over the counter medicines for intestinal disorders containing $(1 \rightarrow 3)$-beta-Dglucan, or administration of blood products, such as albumin and globulin.

Lentinan is clinically used as a biologic response modifier in the treatment of gastric cancer, ${ }^{9}$ and SPG is used in combination with radiotherapy to improve the local response to radiation treatment for cervical cancer. ${ }^{10}$ SPG is isolated from culture media in which the basidiomycete Schizophyllum commune Fries is grown. It is a true glucan (molecular weight approximately 450,000) consisting of repeating units of beta-1,3-D-glycopyranosyl residues. ${ }^{14}$

It has been reported that SPG, upon entry into the body, is transported to the liver, spleen, and mesenteric lymph nodes almost immediately, and its concentration in the blood decreases. ${ }^{15}$ In rats, SPG present in the liver is degraded over a period of six months to SPG-like substances, the molecular weight of which is lower than that of SPG. Furthermore, SPG in the spleen and mesenteric lymph nodes is metabolized at a 
much slower rate than in the liver. Finally, SPG is excreted in the urine, mainly in the form of metabolites with a molecular weight $<10000 .{ }^{16}$ Hase et al studied the clearance of SPG in humans, and found that 30 days after a single intramuscular injection of 20 or $40 \mathrm{mg}$ SPG, the blood SPG concentration was in the order of tens of nanograms per milliliter. ${ }^{17}$ Ishizuka et al reported that lentinan (total dose $400 \mathrm{mg}$ ) and SPG (total dose $280 \mathrm{mg}$ ) can be detected in the body at three years and 350 days, respectively, after the last intramuscular injection. ${ }^{5}$ The biologic half life of $(1 \rightarrow 3)$-beta-D-glucan in humans remains unknown. However, from these reports, it can be concluded that the administration of larger doses of SPG results in elevation of the plasma concentration of $(1 \rightarrow 3)$-beta-D-glucan for longer periods of time.

Our patient was diagnosed with Mycobacterium avium complex pulmonary disease. The specificities of Cand-Tec and Platelia ${ }^{\circledR}$ Aspergillus EIA used to detect fungal infection were $76.9 \%$ and $87.5 \%$, respectively, while their respective sensitivities were $93.9 \%$ and $78.6 \%{ }^{18,19}$ However, deep-seated mycosis cannot be ruled out only on the basis of laboratory data obtained using these kits. In our patient, there was no evidence of fungal infection, including clinical symptoms, radiographic findings, and laboratory data, except for the elevated plasma concentration of $(1 \rightarrow 3)$-beta-Dglucan. Moreover, oral administration of voriconazole, an antifungal agent that acts against a wide variety of yeasts and molds, ${ }^{20}$ for three months did not decrease the plasma $(1 \rightarrow 3)$-beta-D-glucan concentration. We concluded that intramuscular administration of SPG for treatment of cervical cancer 18 years previously had caused the increased beta-D-glucan levels. Thus far, there have been no reports of long-term elevation of blood $(1 \rightarrow 3)$-beta-D-glucan concentrations. The maximum blood concentration of $(1 \rightarrow 3)$-beta-D-glucan in patients with deep-seated mycosis is in the order of several nanograms per milliliter. Thus, we estimated that in our patient, the total amount of $(1 \rightarrow 3)$-beta-Dglucan in circulating blood would be in the order of micrograms..$^{5}$ The cause of the high level of $(1 \rightarrow 3)$ beta-D-glucan in our patient may have been continuous administration of SPG for long periods of time, resulting in the accumulation of hundreds of milligrams of $(1 \rightarrow 3)$-beta-D-glucan.

$(1 \rightarrow 3)$-beta-D-glucan remains in the peripheral blood for many years and interferes with blood $(1 \rightarrow 3)$-beta-Dglucan measurement. Therefore, in patients with cervical cancer, it is important to confirm whether or not SPG has been previously administered before measuring $(1 \rightarrow 3)$ beta-D-glucan levels.

\section{Disclosure}

The authors report no conflicts of interest in this work.

\section{References}

1. Obayashi T, Yoshida M, Mori T, et al. Plasma (1 $\rightarrow 3$ )-beta-D-glucan measurement in diagnosis of invasive deep mycosis and fungal febrile episodes. Lancet. 1995;345:17-20.

2. Williams DL. Overview of $(1 \rightarrow 3)$-beta-D-glucan immunobiology. Mediators Inflamm. 1997;6:247-250.

3. Kakinuma A, Asano T, Torii H, Sugino Y. Gelation of Limulus amebocyte lysate by an antitumor $(1 \rightarrow 3)$-beta-D-glucan. Biochem Biophys Res Commun. 1981;101:434-439.

4. Mori T, Matsumura M. Study on the influence of pretreatment temperature for plasma $(1 \rightarrow 3)$-beta-D-glucan measurement using kinetic turbidimetric Limulus assay and clinical evaluation of $(1 \rightarrow 3)$-betaD-glucan measurement methods. Nippon Ishinkin Gakkai Zasshi. 2000;41:169-176

5. Ishizuka Y, Tsukada H, Gejyo F. Interference of $(1 \rightarrow 3)$-beta-D-glucan Administration in the measurement of plasma $(1 \rightarrow 3)$-beta-D-glucan. Intern Med. 2004;43:97-101.

6. Aketagawa J, Tanaka S, Tamura H, Shibata Y, Saito H. Activation of Limulus coagulation factor $\mathrm{G}$ by several $(1 \rightarrow 3)$-beta-D-glucans: Comparison of the potency of glucans with identical degree of polymerization but different conformations. J Biochem. 1993;113: 683-686.

7. Nagai N, Ohno N, Adachi Y, et al. Application of Limulus test (G pathway) for the detection of different conformers of $(1 \rightarrow 3)$-beta-D-glucans. Biol Pharm Bull. 1993;16:822-828.

8. Ikemura K, Ikegami K, Shimazu T, Yoshioka T, Sugimoto T. Falsepositive result in Limulus test caused by Limulus amebocyte lysatereactive material in immunoglobulin products. $J$ Clin Microbiol. 1989;27:1965-1968.

9. Horiguchi M, Saito M, Sonoda H. [A gastric cancer showing marked improvement (stage IV) with lentinan immunotherapy and 5-FU, MMCchemotherapy]. Gan To Kagaku Ryoho. 1988;15:1973-1977. Japanese.

10. Noda K, Takeuchi S, Yajima A, et al. Clinical effect of sizofiran combined with irradiation in cervical cancer patients: A randomized controlled study. Cooperative Study Group on SPG for Gynecological Cancer. Jpn J Clin Oncol. 1992;22:17-25.

11. Kanda H, Kubo K, Hamasaki K, et al. Influence of various membranes on the plasma $(1 \rightarrow 3)$-beta-D-glucan level. Kidney Int. 2001;60: 319-323.

12. Nakao A, Yasui M, Kawagoe T, Tamura H, Tanaka S, Takagi H. Falsepositive endotoxemia derives from gauze glucan after hepatectomy for hepatocellular carcinoma with cirrhosis. Hepato-gastroenterology. 1997;44:1413-1418.

13. Zhang J, Wang G, Li H, et al. Antitumor polysaccharides from a Chinese mushroom, "yuhuangmo," the fruiting body of Pleurotus citrinopileatus. Biosci Biotechnol Biochem. 1994;58: 1195-1201.

14. Norisuye T, Yanaki T, Fujita H. Triple helix of a Schizophyllum commune polysaccharide in aqueous solution. J Polymer Sci. 1980;18: 547-558.

15. Yadomae T. Structure and biological activities of fungal beta-1, 3-glucans. Yakugaku Zasshi. 2000;120:413-431.

16. Tanji S, Akima K, Horiba M, Amemiya K, Aimoto T. Studies on metabolism and disposition of sizofiran (SPG), an anti-tumor polysaccharide. III. Degradation and excretion of SPG in rats. Yakugaku Zasshi. 1990;110:869-875.

17. Hase T, Ohyama T, Kamasuga T, et al. [Experiments with absorption, distribution, metabolism and excretion of SPG. Kaken Pharmaceuticals Co., Ltd. (Tokyo)] Internal Data. Japanese. 
18. Mitsutake K, Miyazaki T, Tashiro T, et al. Enolase antigen, mannan antigen, Cand-tec antigen, and beta-glucan in patients with candidemia. J Clin Microbiol. 1996;34:1918-1921.

19. Lai CC, Hsu HL, Lee LN, Hsueh PR. Assessment of Platelia Aspergillus enzyme immunoassay for the diagnosis of invasive aspergillosis. J Microbiol Immunol Infect. 2007;40:148-153.
20. Johnson LB, Kauffman CA. Voriconazole: A new triazole antifungal agent. Clin Infect Dis. 2003;36:630-637.

\section{Publish your work in this journal}

The International Journal of General Medicine is an international, peer-reviewed open-access journal that focuses on general and internal medicine, pathogenesis, epidemiology, diagnosis, monitoring and treatment protocols. The journal is characterized by the rapid reporting of reviews, original research and clinical studies across all disease areas.
A key focus is the elucidation of disease processes and management protocols resulting in improved outcomes for the patient.The manuscript management system is completely online and includes a very quick and fair peer-review system. Visit http://www.dovepress.com/ testimonials.php to read real quotes from published authors.

Submit your manuscript here: http://www.dovepress.com/international-journal-of-general-medicine-journal 